\title{
Study on Saponification Technology of Precision Forgings for Constant Speed Transmission Shaft of Car
}

\author{
Wang Delin, Lu Yougen, Wu Bing \\ Manufacturing Engineering Department, Shanghai GKN HUAYU Driveline Systems Co. LTD, Shanghai, China \\ Email address: \\ wangdelin@sds.com.cn (Wang Delin)
}

To cite this article:

Wang Delin, Lu Yougen, Wu Bing. Study on Saponification Technology of Precision Forgings for Constant Speed Transmission Shaft of Car. International Journal of Industrial and Manufacturing Systems Engineering. Vol. 4, No. 1, 2019, pp. 6-9. doi: 10.11648/j.ijimse.20190401.12

Received: November 8, 2018; Accepted: January 30, 2019; Published: February 25, 2019

\begin{abstract}
The high-concentration pickling and the extreme emission and energy consumption of the precision saponification process before the cold finishing of the precision forgings of the constant speed drive shaft of the car. Oil mist emissions from the cold finishing process and relatively harsh working conditions. The purpose of this paper is to develop a new saponification process that saves energy and reduces emissions while ensuring product quality during production. Through a large number of tests, it shows that the saponification of precision forgings for the drive shaft can be completed by three steps including simple saponification liquid spraying, liquid extraction and hot air drying. Thus in this way, the saponification quality is the best, At the same time, it has greatly reduced emissions, while the instability of the saponification quality mainly relates to the control of saponification process.
\end{abstract}

Keywords: Precision Forging Parts, Cold-finishing, Saponification

\section{Introduction}

With the continuous development of human science and technology, the ability to transform nature has undergone tremendous changes. But in the 21 st century, environmental protection has become a top priority for all countries in the world. In the manufacturing sector, companies must not only produce products that do not harm the environment, but also ensure that resources are saved as much as possible during the production process [1-2].

In this context, the car constant speed drive shaft industry began to replace hot forging with warm forging + cold finishing in the 1980s, directly forging the tracks of parts, or leaving only the hard machining allowance. Great savings in energy and materials. After the 1990s, the tooling lubrication in the warm forging + cold finishing process were improved. The synthetic materials were used instead of graphite as the tooling lubricant for warm forging, and the oiling process was used instead of Phosphorus saponification as the cold finishing treatment, greatly reducing emissions and energy consumption [3-5]. In the 21st century, Japan began to explore and improve the original Phosphorus saponification process, eliminating the highly polluted pickling and phosphating process, and developing special environmentally friendly saponified materials to be directly coated on the surface of the workpiece as a cold finishing treatment to reduce dripping. The oil mist is discharged during the oil process and the relatively harsh working environment, and the anti-rusting sequence after the cold forming is eliminated, which saves a lot of energy and reduces emissions. After 2005, the technology was officially used in domestic production in Japan, forming a stable saponification product and rapidly expanding in other industrial fields.

Shanghai GKN HUAYU Driveline Systems Co., Ltd. has 4 automatic precision forging production lines, with an annual output of 20 million precision forgings for the constant speed drive shaft of the car. It summarizes the domestic phosphorus saponification process and draws on the advanced saponification process from abroad to develop a suitable local saponification process. The saponification production line and saponification process of the precision forgings of the constant speed drive shaft of the Shanghai GKN HUAYU Driveline Systems Co., Ltd. are far ahead of the Japanese counterpart in terms of emissions, energy saving and efficiency. It has been developed to become a mature saponification process. In terms of environmental protection, tooling life and production costs have also achieved good results. 


\section{Process Analysis and Production Line Composition}

\subsection{Process Analysis}

As shown in Figure 1, the two types of precision forgings are typical car constant speed drive shaft parts, all of which are medium carbon alloy steels. The shape of the inner cavity is relatively complicated. After the cold forming of the left outer race forgings in Figure 1, the inner cavity surface reaches the final product requirement through a grinding process; in Figure 1, the right Tulip forging is cold forming, and the inner and outer cavity surfaces directly meet the final product requirements. The above two types of forgings require high head dimensional accuracy after cold forming and deformation, and put forward higher requirements for the lubrication treatment of the head before cold forming deformation [6-8].

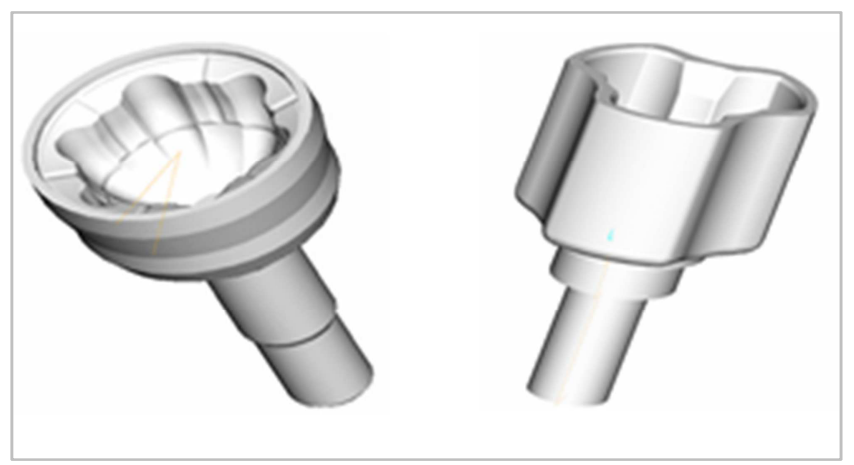

Figure 1. Forgings

\subsection{Production Line Composition}

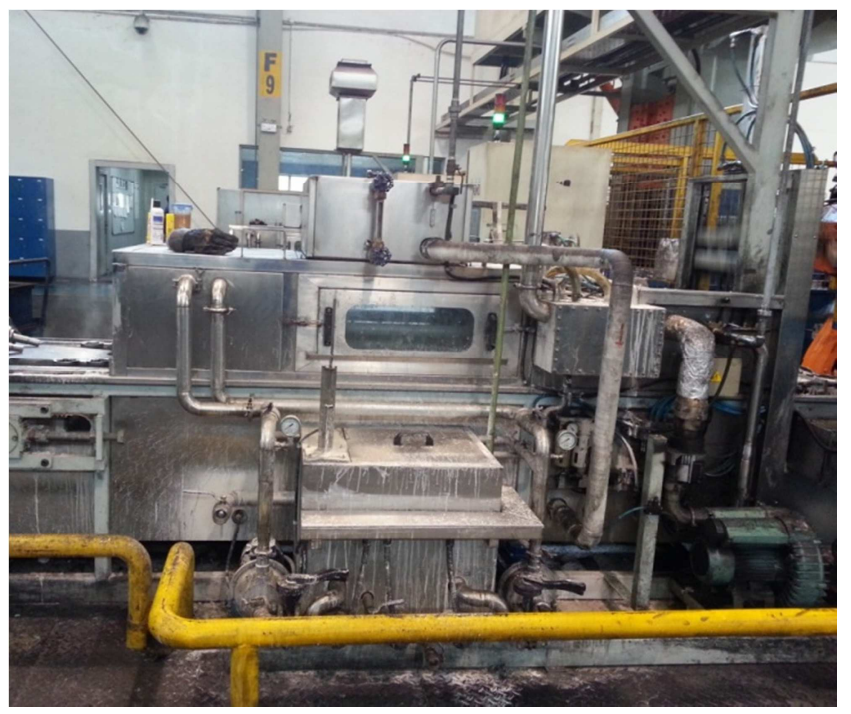

Figure 2. Saponification production line.

As shown in Figure 2, the equipment was developed specifically for the automatic saponification of precision forgings for constant speed drive shafts. Overall equipment size: 4600 (L) X1500 (W) X2340 (H) mm, the overall power is $90 \mathrm{KW}$, working cycle $12 \mathrm{~S} / 2$ pieces. The automatic saponification machine can uniformly apply the saponification liquid to the surface of the warm forging part by continuously stirring the saponification liquid; and the air jet pump is used to drain the saponification liquid in the workpiece, and the precision forging piece does not need to be turned over during the saponification process, so that the whole equipment has a simple structure and low cost. The saponification of the forging surface can be achieved by simply spraying, pumping, and hot air drying in three steps. In the course of use, the saponification liquid is discharged without waste liquid, and the working environment is greatly improved.

\section{Factors Affecting Saponification}

Combined with the application of saponification at home and abroad in the cold forming process and the company's six years of cold forging saponification production experience, the author believes that the following aspects will affect the quality of cold forging saponification.

\subsection{Surface Quality of Precision Forging}

The surface quality of the forging is one of the key indicators to ensure the quality of the saponified film after saponification of the forging. Before the forging is saponified, the surface of the forging should be removed from oil, dust and other impurities. Therefore, the forgings should be shot before saponification until the surface of the forging is metallic [9-11].

\subsection{Concentration}

The concentration of the saponification solution must reach the critical saponification concentration to enter the growth period of the saponified membrane. Moreover, the concentration of the saponification liquid must be uniform, which is advantageous for uniform film formation. Therefore, when cold forming, the force is uniform, which is beneficial to prolong the life of the tooling [12-13].

\subsection{Time}

The weight of the saponified film increases sharply with time, but after the film is completely formed, the saponified film becomes loose and easily falls off. Therefore, the choice of saponification time should be based on actual conditions.

\subsection{Temperature}

The effect of saponification temperature on film formation is very special, unlike general chemical reactions. First, in the middle temperature range $\left(60-70^{\circ} \mathrm{C}\right)$, the saponification increases with temperature by 2 to 3 times $/ 10^{\circ} \mathrm{C}$, and then in the high temperature range $\left(75-90^{\circ} \mathrm{C}\right)$, the saponification reaction drops sharply.

Because a temperature of less than $50^{\circ} \mathrm{C}$, the liquid has a large viscosity and is even cloudy. As the temperature increases, the viscosity decreases, and at $70^{\circ} \mathrm{C}$, it is essentially 
the same as water. Therefore, at about $60^{\circ} \mathrm{C}$, due to the viscosity of the liquid, the saponification process will be controlled by diffusion, and within 4 minutes, the saponification is incomplete [14-15]. However, different saponification components will have different temperature ranges suitable for saponification.

\subsection{PH Value}

When the $\mathrm{PH}$ value is too low, the sodium soap is neutralized and the concentration of the soap is lowered. If the $\mathrm{PH}$ value is too high, the film formation reaction rate is too fast, and the film grains are not closely arranged, resulting in poor appearance and corrosion resistance of the film [16]. Therefore, the effect of $\mathrm{PH}$ value can be attributed to the effect of concentration on saponification. Urban water and purified water as a diluent for the saponification solution will have different effects on the saponification results.

\section{Process Trial}

\subsection{Trial Condition}

A saponification machine having a saponification liquid spray, liquid extraction, and hot air drying function is prepared according to the process requirements, and a saponification liquid, tap water, purified water, a saponification liquid concentration measuring instrument are prepared.

\subsection{Testing Program}

According to the surface quality of the warm forging, the concentration of the saponification solution, the temperature of the saponification solution, the saponification time, the pumping time, the hot air temperature, the $\mathrm{PH}$ value of the water, etc., the saponification treatment before cold forming, and then tracking analysis Forging quality and tooling life.

\subsection{Experimental Procedure}

Spraying - pumping - hot air drying - cold forming.

\subsection{Test Evaluation}

According to the above test scheme, nearly 1 million warm forgings were saponified and cold-formed using different process parameters, and then the surface quality and tooling life of the forgings were tracked and analyzed. The test results show that: tap water as a thinner of saponification liquid, because it contains CL- and other impurities, the forgings are easy to rust after cold forming, and pure water as a thinner of saponification liquid, this problem does not occur; forging surface saponification film after the formation, the saponified film should not be too thick or too thin, the saponified film is too thick, and the saponified film is easily detached, and the saponified film is too thin to achieve a lubricating effect. This test shows that the saponification liquid concentration is 5 to $8 \%$, The saponification film thickness is relatively uniform and the lubrication effect is the best; after the surface of the forging is saponified, the surface must be dry. If the surface of the forging is moist, the saponified film is not completely formed, and thus the lubricating effect is not achieved. The test scheme adopts the hot air of $140 \sim 180^{\circ} \mathrm{C}$ is blown for 5 to 9 seconds, and the saponified film adheres uniformly and is dried to meet the requirements for use.

\section{Process Status and Prospects}

\subsection{Impurity Removal}

In the saponification tank, as the amount of forging saponification increases, the iron blasting and shot blasting particles will increase, which will affect the quality of saponification to a certain extent. A saponification liquid circulation loop is added to the saponification machine, and a magnetic filter is added to the circuit. As shown in Figure 3, In the process of circulating liquid flow, magnetic impurities such as iron blasting and shot blasting particles are adsorbed to ensure the quality of the saponified film.

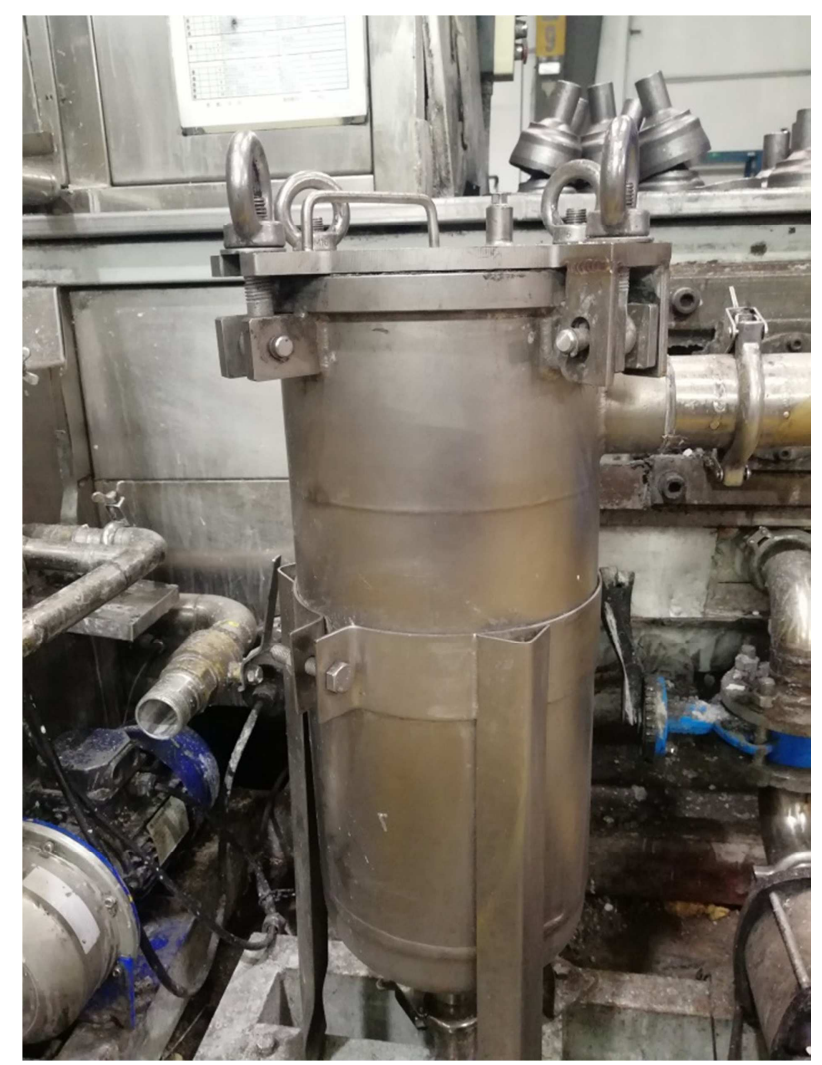

Figure 3. Magnetic filter.

\subsection{Saponification Temperature Control}

The saponification liquid in the saponification tank will decrease correspondingly with the saponification amount of the forging, and the water and the saponification liquid must be added according to a certain ratio, and the water is added by the heated hot water. For the addition of the saponification liquid, there is currently no a good method for heating can only be carried out at room temperature. After the liquid is mixed, the temperature does not reach the working temperature of the saponified mixture. The saponification 
mixture must be heated as a whole and the temperature reaches the temperature required for the work. Work started, efficiency is low, and time is wasted.

\subsection{Rust Prevention}

The development of a new type of saponification liquid, which has the effect of cold forming lubrication and rust prevention of cold forging after the tooling life is not reduced, is the development direction of the new saponification liquid in the next few years.

\section{Conclusions}

The quality of the precision forgings and the unstable tooling life of the precision forgings of the constant speed drive shaft of the car are mainly caused by the uneven thickness of the saponified film. The improvement of the life of the tooling and the reduction of the occurrence of unqualified precision forgings are the key to controlling the quality of the former saponification. Strengthening the control of the saponification process, mastering the correct saponification method and reasonable saponification process is the most effective way to improve the quality of saponification. Using warm forging shot blasting, the concentration of saponification liquid and purified water is $5 \sim 8 \%$, the temperature of saponification liquid reaches $68-75^{\circ} \mathrm{C}$, the saponification time reaches $10 \sim 18$ seconds, the pumping time reaches 5-9 seconds, the hot air temperature reaches At $140-180^{\circ} \mathrm{C}$, this saponification process has been verified by more than 10 million pieces of precision forging saponification. The saponification process can reduce the friction coefficient, improve the life of the tooling, and ensure the quality of precision forgings. Now the saponification process has been successfully applied with a large-scale production of cold forming saponification production line in Shanghai GKN HUAYU Driveline Systems Co., Ltd.

\section{References}

[1] Guo Yinghao, Zhang Yanqiao, Qu Fengzuo, Cui Li, Xu Tongkuan. Study on the Low-polluting zinc phosphating process at med-temperature [J]. Journal of Dalian Polytechnic University, 201130 (1) 54-57.

[2] K. Lange, M. Kammerer, K. Pohlandt, J. Schock. Extrusion technology_Economic manufacturing process of metal precision part [M]. Du Guohui, Zhao Zhen Translation Beijing: China machine press. 2014.

[3] Wang Delin, Lu Yougen, Wu Bing. Application and Development of Warm-Cold Combined Precision Forging Technology Hot Working Technology, 2013, 42 (5) 107-110.

[4] Zhao Zhen, Chen Jun, Wu Gongming. Cold-warm-hot extrusion technology [M]. Beijing: Publishing House of Electronics industry. 2008.

[5] Wang Danian. Principles of metal forming [M]. Beijing: China machine press. 1986.

[6] Chmelz F, Graf von H.-c. Seherr-Thoss, Aucktor E. 3 universal joint and drive shaft $[\mathrm{M}]$. WuDerong, Xiao Shenfa, Tao Jianmin, translation. Beijing: Beijing Institute of Technology Press, 1997.

[7] Wang Delin, Lu Yougen, Wu Bing. Generation and Avoiding of Cracks on Bottom of Tulip during Cold-forming [J] Journal of Netshape Forming Engineering, 2018, 10 (1) 177-180.

[8] Ma huaixian. Metal plastic working principle [M]. Beijing Metallurgical Industry Press, 1997.

[9] Li Weiqi, Ma Qingxian, Developments and Prospects of Friction Behavior during Forging Process [J]. Forging \&Stamping technology, 201439 (6) 9-18.

[10] Li Peng Hu Chengliang Meng Lifen Zhao Zhen. Influence of surface roughness on friction factor in cold forging [J]. Journal of Plasticity Engineering, 200522 (5).

[11] Wen Shizhu, Huang Ping. Principle of Tribology [M]. Beijing Tsinghua University Press, 2012.

[12] Shen Xin, Ouyang Basheng, Yin Xuri. Orthogonal test analysis of pickling process parameters of cold-drawing 45 steel [J]. New Technology and New Technology Press, 2013 (1) 68-70.

[13] Shanghai jiaotong University «Cold extrusion technology» team. Cold extrusion technology $[\mathrm{M}]$. Shanghai: Shanghai people's Publishing house. 1976.

[14] Wang Jiaan, Zhao Zhenfeng, Wang Jialian. Application of phosphating - saponification treatment in low carbon steel cold extrusion process. Forging \& metalforming 2005, (9) 54-56.

[15] Ouyang Basheng, Shen Xin, Yang Yeyao, Yin Xuri. Experimental Analysis of Optimization Parameters of Lubrication Process for Cold-drawing 45 Steel [J]. Hot working technology, 201342 (13) 23-26.

[16] Tang Chunhua. Metal surface phosphating technology [M]. Beijing Chemical Industry Press, 2009. 